\title{
The path to electrical energy using laser fusion
}

\author{
Stephen E. Bodner \\ 546 Carolina Meadows Villa, Chapel Hill, North Carolina 27517, USA \\ (Received 1 November 2019; accepted 7 November 2019)
}

\begin{abstract}
Direct-drive laser fusion has one potential advantage over all other approaches to fusion energy. The hot plasma can be kept near or below the various plasma instability thresholds, if one uses purely spherical targets, with a short wavelength, large bandwidth and optically smoothed excimer laser. Instead of trying to manage laser-plasma instabilities, one avoids them. There is a path to complete the evaluation and development of this energy option, with moderate costs and a moderate time scale. Glass lasers, with their longer wavelength and narrower bandwidth, are no longer useful to evaluate fusion targets.
\end{abstract}

Keywords: excimer laser; laser fusion

\section{Introduction}

In the early 1970s, when the laser fusion programme was in its infancy, several basic physics problems were discovered. When laser beams were focused onto targets, the high laser intensities produced many types of laser-plasma instabilities. A surprisingly large fraction of laser light was either reflected or sent in uncontrollable directions by these laserplasma instabilities. Another surprisingly large fraction of the laser light energy was converted by laser-plasma instabilities into suprathermal electrons; these electrons would have caused preheating of the DT fuel and would have prevented the needed fuel compression to high densities. Also, the laser beams could not produce the illumination uniformity needed for a symmetric implosion. Also, the imploding target shell was much more hydrodynamically unstable than previously predicted; the target shells were now predicted to distort or break during the implosion. The plasma and $\mathrm{X}$-radiation temperatures were also far from the expected thermal equilibrium.

However, over the years these physics problems have apparently all been solved ${ }^{[1]}$. There is now a substantial experimental, theoretical and computational basis for optimism. The preferred solution would use a purely spherical target, illuminated symmetrically by many laser beams ${ }^{[2]}$. The laser would have the shortest possible wavelength, the broadest possible bandwidth and the best possible optical beam smoothing. The preferred laser candidates, and probably the only candidates, are a $\mathrm{KrF}$ or ArF excimer laser.

Correspondence to: S. E. Bodner, 546 Carolina Meadows Villa, Chapel Hill, North Carolina 27517, USA. Email: bodners@icloud.com
In the United States, most of the funds for laser fusion research have been directed toward the use of very complex 'indirect-drive' targets. This programme culminated in the National Ignition Facility (NIF). The failure of the fusion implosions was obvious almost immediately after the NIF laser began illuminating the targets. Little progress has been made in the succeeding years. As a result, there is now some pessimism in the United States about laser fusion. The concern of some is that the computer modeling of fusion targets is not reliable, and that the scientific management has been and remains too optimistic. Thus, an informal proposal for a large programme in symmetric direct illumination of fusion targets for electric power has not had a good reception.

This paper is divided into two parts. First it is necessary to explain why the NIF failed, and why that failure has no relationship to the prospects for symmetric illumination of a spherical target using an excimer laser. The second part of this paper outlines a path toward the development of laser fusion for electrical energy.

Basically, the NIF design was geometrically too complex, and it was designed to operate far above laser-plasma instability thresholds. By comparison, symmetric illumination with excimer lasers of a spherical target is geometrically simple, and it can operate near or below the plasma instability thresholds, because the combination of deep UV and broad bandwidth suppresses the laser-plasma instabilities. Physicists are unlikely to ever be able to predict any plasma behavior when it is an order of magnitude or more above the instability threshold, nor will they be able to accurately calculate laser and plasma behavior in a complex asymmetric 
geometry. However the plasma instability thresholds themselves are all reasonably well understood, both experimentally and theoretically. With symmetric laser illumination one should be able to choose physical parameters that avoid or minimize the laser-plasma instabilities. As Sun Tsu stated: 'To subdue the enemy without fighting is the acme of skill.'

\section{The failure of the National Ignition Facility}

In the early 1970s, Lawrence Livermore National Laboratory began construction of the Shiva glass laser. Their computer model ${ }^{[3]}$ predicted that the symmetric illumination of a small spherical target of pure DT fuel with a few kilojoules of infrared laser light would produce thermonuclear burn and even net energy gain. Their long-term goal was commercial fusion energy. The Shiva laser was supposed to demonstrate this concept. As noted above in Section 1, several basic physics problems were quickly discovered. In 1975, in response to these problems, the Shiva laser, then already under construction, was redesigned for 'indirect drive'. The spherical fusion target would be placed within a cylindrical metal can, with laser entrance holes at the two ends of the can. The prediction was that this indirect, asymmetric illumination would resolve the major physics problems. The poor energy coupling efficiency of indirect drive implied that the original goal of commercial power would have to be discarded, but the prediction was for some fusion success. Instead, the indirect-drive design also failed. To try to solve the various physics problems, the indirect-drive target was modified several times over the succeeding decades. Each modification has made the target more complex: adding gas fills; using two cones of laser beams with separate power tuning; changing the shape of the cans; changing the heavy metals used for the can wall; using different ablator materials.

These variations in indirect-drive target designs still faced the same two basic problems. First, their laser illumination was initially asymmetric: the laser illumination was designed to directly heat the inside of the can along a set of rings, producing soft X-rays. The X-radiation flow from those hot rings was predicted to then lead to a nearly symmetric illumination of soft X-rays on the spherical target. It worked on their computer! But the real three-dimensional plasma flows, the real laser propagation, the real non-LTE hot plasmas, the real non-LTE X-radiation transport, and the real laser-plasma instabilities, are in fact too complex to ever be able to calculate with sufficient precision. The second basic problem was the focusing of all of the laser energy through the two entrance holes in the ends of the can. This focusing maximized the laser intensity. The laser light was then propagated long distances in the can through a hot plasma. This frontal attack, using high laser intensities and long plasma path lengths, is a prescription for the worst possible laser-plasma instability problems. And of course it was impossible to sufficiently diagnose the behavior of the laser propagation and the hot plasma inside the metal can, so they could not be certain where all of the laser energy was actually deposited, or how the plasma within the can actually evolved. Also early-time low-mode asymmetries could not be corrected by using the opposite low-mode asymmetry later in the laser pulse. They would have to somehow measure the time-dependent asymmetries, and somehow measure the many sources of the asymmetry inside the can. The detailed physics reasons for the failure of the NIF had actually been predicted before construction began ${ }^{[4]}$.

Various other non-symmetric laser fusion concepts have been proposed and investigated in recent decades. In the 'fast-ignition' concept, a spherical target is first symmetrically imploded to high density, but without a central spark plug. The burn is then initiated by heating one side of the imploded compressed sphere with a separate high-intensity, short-pulse laser. One variant used a physical cone in the target to guide spark plug formation. These alternate fusion concepts $^{[5]}$ all suffer from the same two basic problems as indirect drive: the geometries are too complex to calculate, and they require utilizing and controlling laser-plasma instabilities instead of just trying to avoid them.

\section{Glass lasers are not relevant to an evaluation of laser fusion}

With the very large financial investment that has already been made in glass lasers, some people have hoped that the NIF could eventually be modified and used to evaluate symmetric illumination designs. That hope is not consistent with the science.

An ArF laser should be able to produce a bandwidth of $5 \mathrm{THz}$. Calculations, with various supporting experiments at narrower bandwidths, indicate that this $5 \mathrm{THz}$ bandwidth, along with the short wavelength of ArF, and with optical beam smoothing, should suffice to control the deleterious laser-plasma instabilities. A $\mathrm{KrF}$ laser has a bandwidth of $3 \mathrm{THz}$. This bandwidth is more marginal, but may also suffice. And by using very limited amounts of forward rotational Raman scattering in a gas, it may be possible to enhance the $3 \mathrm{THz} \mathrm{KrF}$ bandwidth ${ }^{[6]}$.

The NIF glass laser bandwidth is limited to $0.27 \mathrm{THz}$ after conversion to the third harmonic. This is at least a factor of ten less than what is probably needed for laser fusion. The Omega glass laser has previously operated at up to $1.0 \mathrm{THz}$, but there were focusing problems associated with this bandwidth. The Omega glass laser bandwidth now operates at $0.33 \mathrm{THz}$.

There is a current suggestion ${ }^{[7]}$ to improve glass lasers by increasing the bandwidth through the use of an optical parametric amplifier, followed by some unspecified broadband 
frequency conversion. This recent suggestion is still in its early stages of development. If successful, the bandwidth conversion would hopefully not lead to the same problems in the focal plane as was found with the $1 \mathrm{THz}$ version of Omega.

Even if the bandwidth limitation to glass lasers could somehow be solved, there are other fundamental limitations to the use of the NIF. The NIF beam-to-beam energy balance is poor, and the NIF focal quality with their current optical beam smoothing is poor. We can conclude that it is very unlikely that the NIF will have any value in any future evaluation of spherical targets using symmetric illumination. The continued expenditure of significant funds on glass lasers by the United States in the hope of someday obtaining ignition with the NIF, while refusing to fund any further development of the much lower-risk excimer laser, is a beautiful example of the 'sunk cost' logical fallacy.

\section{Ignition is the wrong intermediate programme goal}

Ignition in the fuel occurs when the alpha particles produced by the thermonuclear burn are stopped within the alreadyhot DT plasma, further increasing its temperature. This self-heated plasma becomes a spark plug. A thermonuclear burn wave then spreads from the hot spark plug into the surrounding cold, compressed DT fuel. Since it takes much less laser energy to compress DT than it does to heat DT to burn temperatures, the overall target energy gain becomes much higher once the laser energy exceeds ignition threshold and the burn can propagate into the cold fuel. Also, the fractional fuel burnup increases with increasing amounts of cold compressed fuel once the target is above ignition. Thus the physics importance of ignition.

A common but mistaken view is that the next step toward commercial fusion energy should therefore be a demonstration of ignition. In this view, after ignition is achieved the higher fusion energy gains needed for commercial electric energy would then be obtained by increasing the laser energy, with perhaps also some unspecified optimization of the target design.

But the above story is not scientifically correct. Consider the function $G\left(E_{L}\right)$, where $G$ is the energy gain of the fusion target and $E_{L}$ is the laser energy. Plotting the function $G$ versus $E_{L}$, it has a value much less than one when the laser energy is below ignition. Near ignition the gain curve starts to rise rapidly. Well above ignition, the function $G$ rises more slowly, increasing as the laser energy compresses more cold DT fuel. The problem is that at larger laser energies, well above ignition, the actual value of $G$ is dependent upon the overall efficiency with which the laser energy is converted to compressed DT fuel, and also on the overall efficiency of the thermonuclear burn. If either efficiency is too low, then the energy gain $G$ will never reach the high value that is needed for economically viable electric power. Success with ignition does not necessarily imply that sufficient energy gain can ever be achieved using reasonable laser energies.

Instead of ignition, a better intermediate goal would be sufficient energy gain such that one can easily extrapolate to the higher energy gain needed for electric power. This revised goal incorporates the real inefficiencies of compression and burn. Ignition as an intermediate goal is too easy with symmetric illumination.

\section{The primary physics requirements for laser fusion energy}

The previous decades of experimental and theoretical research imply that there are several simultaneous requirements on a successful laser fusion implosion.

- The laser frequency must be in the ultraviolet, with a laser wavelength preferably in the range from $0.25 \mu \mathrm{m}$ $(\mathrm{KrF})$ to $0.19 \mu \mathrm{m}(\mathrm{ArF})$.

- The laser must have a bandwidth of at least 3 to $5 \mathrm{THz}$ ( $\mathrm{KrF}$ and $\mathrm{ArF}$ ).

- The laser must be optically smoothed, with a broad bandwidth, to produce sufficient laser beam focal uniformity.

- The laser focal profile should be adjustable, and zoom inward during the implosion, to roughly match the size of the spherical target as it spherically converges, and to maintain the uniformity of pressure.

- The spherical ablator surrounding the DT fuel should be a low-density $\mathrm{CH}$ foam, wicked with frozen DT, and surrounded by a very thin metallic coating, probably gold plus palladium.

- The temporal laser pulse shape should start with a few short laser pulses, followed by a carefully tuned rising pulse.

There are more requirements that could be listed, but here are the reasons for the above list. The short laser wavelength leads to better collisional absorption of the laser energy, at higher plasma densities. With absorption at higher plasma density, there is less risk of plasma instabilities, and there is also a higher rocket efficiency. Also, the higher pressure generated with higher density absorption allows for thicker target shells with reduced Rayleigh-Taylor fluid instability growth. If the laser bandwidth is greater than the growth rate of a plasma instability, then the bandwidth reduces or eliminates the instability ${ }^{[8-10]}$. This bandwidth improvement is most effective if the laser wavelength is short, because then the plasma instability growth rate is also minimized and the laser intensity is minimized. Optical smoothing with induced spatial incoherence $^{[11]}$ (ISI) can produce the time-averaged 
laser uniformity that is needed for a spherical implosion, thus avoiding shell destruction from fluid instabilities. Optical smoothing with ISI also prevents optical self-focusing of the laser energy in the plasma corona, thereby reducing the risk of plasma instabilities ${ }^{[12]}$. Optical smoothing with ISI may also provide an easy flexibility to adjust the size and shape of the focal profile during the implosion, to match the imploding spherical target ${ }^{[13]}$. The low-density $\mathrm{CH}$ foam ablator wicked with DT is currently believed to be necessary to avoid excessive soft X-ray generation. If the ablator has too much carbon, then during the thermonuclear burn the heated carbon is calculated to emit enough soft X-rays to melt the inner wall of the chamber. An ablator that is mostly DT with only a small percentage of carbon prevents melting of the chamber wall. Also, the lower $\mathrm{Z} / \mathrm{A}$ of tritium enhances the rocket efficiency. The ultrathin coating of metal is needed to protect the frozen DT from preheating during the injection phase into the hot chamber, and to allow for DT permeation during target manufacturing. The metal coating also provides an earlytime burst of soft $\mathrm{X}$-rays that radially tunes the adiabat of the ablator, thereby helping to reduce the fluid instabilities ${ }^{[14]}$. The carefully tuned temporal laser pulse shape is needed not only to produce a low-isentrope implosion, but to also help tune the radial adiabat of the ablator to minimize the fluid instability ${ }^{[15-19]}$, and to minimize the initial mass imprint from laser nonuniformities ${ }^{[20]}$.

For the research scientists who use glass lasers, most of the above list of requirements is a daunting set of major challenges that they are still trying to solve. Their laser frequency is too low, their bandwidth is too narrow, and their optical smoothing method is inferior because of residual longwavelength nonuniformities. But an ArF excimer laser with ISI smoothing should meet the above laser requirements ${ }^{[21]}$. Perhaps also a KrF laser ${ }^{[22]}$.

\section{A diode-pumped solid-state laser does not meet the requirements for laser fusion energy}

There is no application for glass lasers in a fusion power plant because of its very low efficiency, high optical damage, high cost, etc. Some people have hoped that some of the science and technology of glass lasers could eventually be applied to diode-pumped solid-state lasers (DPSSLs). But DPSSLs are narrow bandwidth lasers. To produce the needed large laser bandwidth, there is a concept ${ }^{[23]}$ to build a very large number of DPSSLs, each with its own different central frequency, each then converted to the third harmonic. Each small DPSSL would illuminate a different small section of a final focusing mirror, so that each mirror would, in total, contain several $\mathrm{THz}$ of bandwidth. All parts of the focal spot would receive the full bandwidth from the entire focusing mirror. The proponents of this idea claim that the target will then have the same or even larger bandwidth than can be obtained from an excimer laser.

Aside from the complexity and cost of using tens of thousands of parallel DPSSLs through the entire laser chain, with different central frequencies, and perhaps requiring the development of new types of DPSSLs, there are also some fundamental target physics questions. Would the combination of laser beams produce sufficient spatial uniformity in the focal plane, even though each individual DPSSL would not have any optical beam smoothing? Also, each location on the final focusing mirror would be illuminated by just one of the DPSSLs, with its relatively narrow frequency. That implies that in the target plane each frequency component would be associated with a narrow angle of propagation vectors. Would the small filled region of combined frequencyvector space enjoy the same advantages for the target physics as an excimer laser that fills a much broader region of frequency-vector space? Is the lack of zooming important? Would the lower mean laser frequency of a DPSSL suffice to control all of the laser-plasma instabilities? My own view is that there is a negligible prospect of using DPSSLs for commercial fusion power.

\section{The path to electric power using laser fusion}

Most physicists view fusion as currently a physics problem. In this view, if our evolving physics knowledge requires future changes in the design, then detailed engineering would be premature. If the physics does not work, then why worry now about the engineering?

But for laser fusion, with its physical separability of components, there can be a different viewpoint. Why bother evaluating the physics target performance if it is not possible to build a laser that meets all of the current requirements of efficiency, beam uniformity, bandwidth, frequency, capital and operating costs, reliability, durability and pulse shaping? Why bother imploding a target with an expensive and carefully grown single-crystal piece of DT, if in an actual manufacturing plant, with high target production volumes, the DT ice crystalizes with too much mass nonuniformity for a symmetric implosion? If laser fusion is going to fail due to laser problems or target fabrication problems, would not it be cheaper and faster to discover this sooner rather than after an expensive fusion test?

For magnetic fusion, with its tightly integrated geometry, perhaps the physics-first approach is the only approach. But for laser fusion, with its physically separate target factory, laser and chamber, a parallel development should be the preferred approach. Instead of first building expensive flagship fusion facilities that only demonstrate high yield with single laser pulses, in a business approach one would first develop and evaluate the highest-leverage key issues that require the least funding. 
Shortly before my retirement I proposed, obtained initial funding and initiated a programme in the United States called High Average Power Lasers (HAPL). This was a nation-wide programme, eventually involving more than 30 institutions in the United States, and managed by the U.S. Naval Research Laboratory. It was an integrated programme to develop the science and technology basis for electric power generation using laser fusion with symmetric illumination. The programme emphasis was on experimental verifications, not computer modeling. A large team of scientists and engineers throughout the United States developed and tested credible approaches to high-repetition-rate excimer lasers, final optics, target fabrication, target injection and engagement, chamber technologies, tritium processing, vacuum systems and laser maintenance ${ }^{[24]}$. Although this programme was very successful, it lost its funding prematurely.

Stage One of a new laser fusion energy programme would be to complete the long-term objectives of the HAPL programme. It would include the development and testing of just one line of laser amplifiers of $\mathrm{ArF}$ and/or $\mathrm{KrF}$ lasers, that could operate reliably at one pulse per minute, and that would meet all of the other above requirements of efficiency, beam quality, etc. This first stage would also include building a target factory that could produce one target per minute that meets all of its commercial requirements. The rate of one pulse per minute for the laser and target factory has been chosen for Stage One, instead of the final goal of approximately five pulses per second, to delay the expenses of tritium recycling and heat removal. Tritium recycling and heat removal would be expensive to develop, but are probably not fundamental problems, and thus could be addressed at a later programme stage. The first stage would also include a detailed optical design for an implosion facility, and would demonstrate control of optical damage from the short laser wavelength. There would also be detailed computer studies of laser propagation through the target corona, to more accurately determine the specific time-dependent requirements on the laser focal profile for a symmetric implosion. Along with the computer studies, there would be a new excimer laser-target facility, an enhancement of the Nike KrF laser ${ }^{[25]}$ with more than $20 \mathrm{~kJ}$ laser output. This facility, using planar or near-planar targets, would have the laser focal intensity and pulse duration to complete the validation of the thresholds of the various laserplasma instabilities.

After Stage One has been fully successful, one would begin Stage Two: construction of an integrated one-pulseper-minute laser fusion implosion facility, using enough laser energy to ensure that the targets would have significant energy gain. This facility would be used to validate and optimize the fusion target design, and then to test and optimize the chamber wall materials.

After Stage Two has been fully successful, one would begin Stage Three: an engineering test facility with 5 pulses per second that could handle tritium recycling, laser and chamber heat removal, electric power generation, etc.

Proceeding in stages, with each stage having to be proven successful before beginning the next stage, might appear to be an unnecessarily slow approach to fusion energy. But past experience suggests that it is faster to fully verify each step first, rather than spend time and money later trying to fix mistakes from hasty and incorrect decisions driven by some politically motivated schedule.

\section{Summary}

The original laser fusion concept of symmetric illumination of spherical targets is now very attractive. In spite of very limited funding, the scientists in this programme have apparently solved all of the key fundamental physics problems, and there is a clear path in stages to commercial power. There is still a risk of failure, but the promise is now so great that the concept merits a larger investment and evaluation. The lowest risk approach would use a laser with the shortest possible wavelength, the broadest possible bandwidth and with the optimum optical smoothing methods; in other words, an excimer laser. The failure of the NIF glass laser is irrelevant to this fusion approach.

\section{Acknowledgements}

The author is retired and did not receive any funding from any source to prepare this paper.

\section{References}

1. R. S. Craxton, K. S. Anderson, T. R. Boehly, V. N. Goncharov, D. R. Harding, J. P. Knauer, R. L. McCrory, P. W. McKenty, D. D. Meyerhofer, J. F. Myatt, A. J. Schmitt, J. D. Sethian, R. W. Short, S. Skupsky, W. Theobald, W. L. Kruer, K. Tanaka, R. Betti, T. J. B. Collins, J. A. Delettrez, S. X. Hu, J. A. Marozas, A. V. Maximov, D. T. Michel, P. B. Radha, S. P. Regan, T. C. Sangster, W. Seka, A. A. Solodov, J. M. Soures, C. Stoeckl, and J. D. Zuegel, Phys. Plasmas 22, 110501 (2015).

2. S. E. Bodner, A. J. Schmitt, and J. D. Sethian, High Power Laser Sci. Eng. 1, 2 (2013).

3. J. Nuckolls, L. Wood, A. Thiessen, and G. Zimmerman, Nature 239, 139 (1972).

4. S. E. Bodner, https://www.academia.edu/6453024/Bodner_ comments_on_NIF_1995.

5. M. Tabak, D. Hinkel, S. Atzeni, E. M. Campbell, and K. Tanaka, Fusion Sci. Technol. 49, 254 (2006).

6. J. Weaver, R. H. Lehmberg, S. P. Obenschain, D. Kehne, and M. Wolford, Appl. Opt. 56, 8618 (2017).

7. D. H. Froula, http://www.firefusionpower.org/Froula-FPA2018-V6.pdf.

8. J. J. Thomson, Nucl. Fusion 15, 237 (1975).

9. S. P. Obenschain and N. C. Luhmann, Appl. Phys. Lett. 30, 452 (1977).

10. J. W. Bates, J. F. Myatt, J. G. Shaw, R. K. Follett, J. L. Weaver, R. H. Lehmberg, and S. P. Obenschain, Phys. Rev. E 97, 061202(R) (2018). 
11. R. H. Lehmberg and S. P. Obesnchain, Opt. Commun. 46, 27 (1983).

12. A. J. Schmitt, Phys. Fluids 31, 3079 (1988).

13. R. H. Lehmberg and J. Goldhar, Fusion Technol. 11, 532 (1987).

14. S. E. Bodner, D. G. Colombant, A. J. Schmitt, and M. Klapisch, Phys. Plasmas 7, 2298 (2000).

15. S. P. Obenschain, D. G. Colombant, M. Karasik, C. J. Pawley, V. Serlin, A. J. Schmitt, J. L. Weaver, J. H. Gardner, and L. Phillips, Phys. Plasmas 9, 2234 (2002).

16. M. Karasik, J. L. Weaver, Y. Aglitskiy, J. Oh, and S. P. Obenschain, Phys. Rev. Lett. 114, 085001 (2015).

17. V. N. Goncharov, J. P. Knauer, P. W. McKenty, P. B. Radha, T. C. Sangster, S. Skupsky, R. Betti, R. L. McCrory, and D. D. Meyerhofer, Phys. Plasmas 10, 1906 (2003).

18. T. J. B. Collins, J. P. Knauer, R. Betti, T. R. Boehly, J. A. Delettrez, V. N. Goncharov, D. D. Meyerhofer, P. W. McKenty, S. Skupsky, and R. P. J. Town, Phys Plasmas 11, 1569 (2004).

19. K. Anderson and R. Betti, Phys. Plasmas 11, 5 (2004).

20. N. Metzler, A. L. Velikovitch, and J. H. Gardner, Phys. Plasmas 6, 3283 (1999).

21. S. P. Obenschain, firefusionpower.org/Obenschain $\% 20 \mathrm{NRL} \%$ 20\%20FPA-2018\%20.pdf.

22. J. L. Weaver, J. Oh, L. Phillips, B. Afeyan, J. Seely, D. Kehne, C. M. Brown, S. P. Obenschain, V. Serlin, A. J. Schmitt, U. Feldman, R. H. Lehmberg, E. McLean, and C. Manka, Phys. Plasmas 20, 022701 (2013).
23. D. Eimerl, E. M. Campbell, W. F. Krupke, J. Zweiback, W. L. Kruer, J. Marozas, J. Zuegel, J. Myatt, J. Kelly, D. Froula, and R. L. McCrory, J. Fusion Energy 33, 476 (2014).

24. J. D. Sethian, D. G. Colombant, J. L. Giuliani, R. H. Lehmberg, M. C. Myers, S. P. Obenschain, A. J. Schmitt, J. Weaver, M. F. Wolford, F. Hegeler, M. Friedman, A. E. Robson, A. Bayramian, J. Caird, C. Ebbers, J. Latkowski, W. Hogan, W. R. Meier, L. J. Perkins, K. Schaffers, S. A. Kahlik, K. Schoonover, D. Sadowski, K. Boehm, L. Carlson, J. Pulsifer, F. Najmabadi, A. R. Raffray, M. S. Tillack, G. Kulcinski, J. P. Blanchard, T. Heltemes, A. Ibrahim, E. Marriott, G. Moses, R. Radell, M. Sawan, J. Santarius, G. Sviatoslavsky, S. Zenobia, N. M. Ghoniem, S. Sharafat, J. El-Awady, Q. Hu, C. Duty, K. Leonard, G. Romanoski, L. L. Snead, S. J. Zinkle, C. Gentile, W. Parsells, C. Prinksi, T. Kozub, T. Dodson, D. V. Rose, T. Renk, C. Olson, N. Alexander, A. Bozek, G. Flint, D. T. Goodin, J. Hund, R. Paquio, R. W. Petzoldt, D. G. Schroen, J. Sheliak, T. Bernat, D. Bittner, J. Karnes, N. Petta, J. Streit, D. Geller, J. K. Hoffer, M. W. McGeoch, S. C. Glidden, H. Sanders, D. Weidenheimer, D. Morton, I. D. Smith, M. Bobecia, D. Harding, T. Lehecka, S. B. Gilliam, S. M. Gidcumb, D. Forsythe, N. R. Parikh, S. O'Dell, and M. Gorensek, IEEE Trans. Plasma Sci. 38, 690 (2010).

25. S. P. Obenschain, R. H. Lehmberg, D. Kehne, F. Hegeler, M. Wolford, J. D. Sethian, J. Weaver, and M. Karasik, Appl. Opt. 54, 103 (2015). 